\title{
A seven-factor procedural analysis of safety culture known measurement: A case study at KLM E\&M
}

\author{
Dr Adel A. Ghobbar ${ }^{\dagger}$ \\ Mr Faouzi Boutahri \\ Prof. Richard Curran \\ Delft University of Technology, Faculty of Aerospace Engineering, Aerospace Management and \\ Operations Department, Kluyverweg 1, 2629 HS Delft, The Netherlands
}

\begin{abstract}
This paper present the result of a safety culture questionnaire within aircraft maintenance engineering, initially produced by Diaz-Cabrera et al. (2007) was developed and validated leading to a better result of seven factors. This safety culture questionnaire (SCQ) is further used as a tool to do a pilot assessment on the factors retrieved. The report ends with the implementation of literature distilled concepts about safety culture including the validated practical safety assessment tool leading to a complete SMS (Safety Management System) which is both reactive as proactive.
\end{abstract}

To increase aviation safety it is compulsory, since the beginning of 2007, to report occurrences to the quality control. This directive is legislation on occurrence reporting in civil aviation from the European Union. The Occurrence reporting process was also regulated before by EASA Part 145.A.60 although their specific purposes seem to differ.

The purpose of the EASA set of regulation and the purpose of the EC regulation are different. The purpose of the EC regulation is to require Member States to put a system in place and to exchange data using the ECC-AIRS tool. The purpose of the EASA regulations on reporting is to ensure continuing airworthiness. It should be noted that the list of reportable occurrences are consistent between the EASA texts and the EC regulation. Therefore there are no plans to harmonize these requirements in a single one (EASA, 2006)

Part A.60: "the organization shall report to the competent authority, the state of registry and the organization responsible for the design of the aircraft or the component. There is therefore no direct reporting to the Agency".

Although the purposes seem to differ because the EC-directive is primarily for benchmarking with other EU member states and the EASA regulation is to ensure

\footnotetext{
† Email: A.A.Ghobbar@tudelft.nl
} 
continuing airworthiness they have one thing in common: an occurrences reporting system (ORS).

KLM E\&M has an occurrences reporting system in place since 2003. An occurrence reporting system gives information on safety issues in maintenance practices. Errors, violations and misconduct result occurrences that are reported and consequently solved within the maintenance unit. This should ensure that the organization becomes resilient to similar errors in the future.

The average number of reported occurrences a year is nearly a thousand, on the one hand one may presume that the safety performance is good but the USO has detected frustrations in the occurrences reporting system (ORS). These frustrations are shown by a number of reports which were reported in the ORS but don't qualify as an occurrence according to the examples given in the directive EU 2003/42/EC.

This research has been written at the support unit of the Line Maintenance department. The support unit consists of three project managers and one assistant project manager, respectively in the domain of: communication, enterprise performance system \& safety management. The research was done within the support unit, domain safety management. The project manager safety management also has the function of unit safety officer (USO). This means that the project manager safety management is both a developer of the process as a key player in the total safety management system within Engineering \& Maintenance. Due to these roles the project manager safety management has an assistant safety management.

The initial research objective was composed after input from KLM E\&M; improving the safety performance by implementing the concept of safety culture and developing and testing a practical tool for the assessment of safety culture which unmasks crucial latent factors and monitors improvement. From the research objective three research questions were distilled:

- What is the quality of the feedback given in occurrence reports?

- Is there a relationship between constructive feedback and motivation in reporting?

- Identify key issues for a good occurrences management system?

- What are the key features of a good safety culture?

- What is the relationship between safety culture and safety management system (SMS)?

The researcher had done a previous literature study on human factors and as such he developed an expertise in the field of human factors and their root causes. As part of the research he held interviews with various maintenance engineers and management. $\mathrm{He}$ realized that an important concept was missing in the current safety management system, namely: Safety Culture.

Keywords: Safety Culture; Human Factors; Maintenance Error; Aircraft Maintenance.

\section{Introduction}


Since the beginning of 2007, the European Commission (EC) has enforced the reporting of safety occurrences in civil aviation organizations to the competent authorities, in order to increase safety. The airline operator at which this research study was performed (henceforth referred to as Operator X) has an Occurrence Reporting System (ORS) in place since 2003. On average, there are approximately one thousand reported occurrences at Operator X per year. Based on this number and the size of operations at Operator X, one may presume that reporting performance is above industry average. However, safety staff has voiced frustrations about the ORS. One example of this phenomenon is recurrent reporting of the same occurrence by a maintenance engineer because he felt that nobody was taking his report seriously. The prevalence of these types of frustrations with the ORS has been acknowledged by senior management staff and was attributed to the poor quality of feedback given upon occurrence reports. From this premise, the pragmatic goal of this research was to develop the answer to the question "How can we keep the submitters of occurrence reports satisfied so that they keep submitting?"

Based on this question the following research questions were composed:

- What is safety culture, define this concept?

- What is the relationship between safety culture and safety management system (SMS)?

- What are the key features of a good safety culture?

- Is there any evidence that a good safety culture is indicative for the state of safety like a correlation with accident rates?

- How can safety culture be measured?

\section{Methodology}

To answer the research questions in a systematic manner, the research has been structured according to the research model of Kempen and Keizer (Kempen and Keizer 2000). It's outlined structure was used as a guideline for the conducted research. Kempen \& Keizer's research model consists of three phases:

- The orientation phase

- The research phase- and the phase in which the solution is found

- The implementation phase

Error! Reference source not found. 1 shows the outlined structure of this research model.

Table 1: Research model (based on Kempen and Keizer, 2000)

\begin{tabular}{|l|l||}
\hline Phase & Research Problem \\
\hline Orientation phase & $\begin{array}{l}\text { Internal and External Orientation } \\
\text { Literature study: Safety Culture }\end{array}$ \\
\hline $\begin{array}{l}\text { The research phase-and the phase in } \\
\text { which the solution is found }\end{array}$ & $\begin{array}{l}\text { Developing a practical tool for the } \\
\text { assessment of safety culture } \\
\text { Validating the tool: pilot assessment }\end{array}$ \\
\hline
\end{tabular}


The orientation phase consisted of several meetings with safety staff, as well as maintenance staff within the organization. Also internal documents about working procedures, regulations and work process information were studied to obtain a clear image of the situation.

From this literature study the most suitable method and model for assessing safety culture was chosen and adapted for this particular organization. The results of the pilot assessment will be discussed.

The practical tool for the assessment of safety culture will be implemented in the ORSprocess as well as the key issues as conducted by the literature study. This phase also consists of integrating the results of the pilot assessment in the recommendations for the ORS and the feedback model.

\section{Defining safety culture}

\subsection{Definitions}

The commitment of management to safety and the norms and values of personnel are part of what is called "Safety Culture". It is only one aspect of the broad concept of "organizational culture" (Wiegmann, Zhang et al. 2002).

To fully comprehend this concept of "safety culture", the term must be defined more closely. A total of 26 definitions from different industries have been compared by Wiegmann (Wiegmann, Zhang et al. 2002), Guldenmund (Guldenmund 2000) and Choudry (Choudry, Dongping et al. 2007) to determine the commonalities between them. Wiegmann has determined seven commonalities between the definitions which hold for the extended comparison done in this research (Wiegmann, Zhang et al. 2002). These commonalities shape the outline of safety culture and are the following:

1. Safety culture is defined at the group level or higher, which refers to the shared values among all the members within a group or organization.

2. Safety culture deals with formal safety issues in an organization, and is closely related to, but not restricted to, the management and supervisory systems.

3. Safety culture emphasizes the contribution from the top to the work floor within an organization.

4. Members' behavior at work is impacted by the safety culture of an organization.

5. Safety culture is usually reflected in the contingency between reward systems and safety performance.

6. Safety culture is a learning culture, willing to develop and learn from errors, incidents, and accidents.

7. Safety culture is relatively enduring, constant entity and resistant to change. 


\subsection{Safety culture models}

Extending the work of Reason (Reason 1998), Hudson has developed a list of five components working together to form a safety culture (Hudson 2001). These components are:

- Informed culture: managers need to know what goes on in their organization and can only be accomplished if employees report their mistakes.

- Just culture: employees will only report their mistakes if they do not feel threatened. However, there must also be accountability for committed actions.

- Flexible culture: the organization can react promptly to change.

Learning culture: organization should learn from its mistakes by providing feedback (control) and improve by actions

Wary culture: this characteristic was added by Hudson and features pro-activeness as well the idea of remaining "wary".

Furthermore, Hudson identifies 5 stages of maturity through which an organization evolves. These are: pathological, reactive, calculative, proactive and generative (Hudson 2001). Another model, offered by Lafreneire, identifies the relationship between safety culture and the safety management system (SMS) (Lafrenière 2006). It considers safety culture to be at the heart of an organization, while the SMS is the muscle (safety rules, procedures, processes, regulations, documentation, etc.) within the organization. An organization with people willing to work safely but which does not have any formalized framework will not lead to positive safety outcomes. The same holds for any organization which has clear processes and regulations, but no employees that believe in safety.

\subsection{Features of a "good safety culture"}

From the studies performed by the International Civil Aviation Organization (ICAO,1992) and by the Health and Safety Executive (HSE 2002), three main features can be determined which are considered to provide a healthy safety culture:

- Management commitment to safety: intentions, words and deeds

- Employee commitment to safety: this awareness can be fostered through training, workshops and employer encouragement.

- Multi-layer Communication: Communication efficiency between employees, employees and management and between management of different levels are encouraged.

\section{Measuring safety culture}

There is an ongoing discussion whether or not an abstract concept like safety culture can be measured objectively. Although such a discussion might hold great academic value, it is of little practical relevance for the industry which is need of tangible guidelines. Glendon and McKenna have suggested that components of safety culture are observable. Thus, by applying qualitative as well as quantitative study methods to these observable 
components of safety culture, the best possible picture can be composed (Glendon, McKenna et al. 2006).

Understanding the sum of behavior and motivation of all employees is considered essential to assess and improve the safety culture of an organization. Therefore, a questionnaire was designed to provide a measure of the visible components of safety culture within the organization.

The themes to be studied in the questionnaire were determined based on verbal interviews with senior safety staff. Their main concerns can be summarized as follows:

- Problems with the current occurrences reporting system: feedback, investigation

- Top-management and line-management preference of achieving operational goals at the expense of safety procedures

- Rewarding and complimenting the submitters of occurrence reports

- Lack of safety-related training

- Inefficiency of communication on and between different levels

These themes were largely found present in a quantitative Likert-scale-based questionnaire developed by Diaz-Carbera (Diaz-Cabrera, Hernandez-Fernaud et al. 2007). To further complete this questionnaire, a number of qualitative questions were added. These questions were partly based on literature and partly on the interviews with senior safety staff.

The questionnaire was then distributed to 209 certified maintenance engineers.

\section{Multi-Taxonomy Classification Systems within Airline Operators}

Aircraft maintenance organizations face maintenance errors resulting in occurrences every day. Technology advances but still the most sophisticated processes in aircraft maintenance rely on human judgment and skills and an unchangeable part of the human condition is fallibility. Add to this the fact that maintenance tasks are often performed in an environment with time pressures, sparse feedback, and difficult ambient conditions, result in the fact that maintenance organizations attract far more than their fair share of human performance problems (Reason and Hobbs, 2003).

Maintenance errors affect both flight safety and bottom line results. Studies show that maintenance errors are involved in $14-16 \%$ of all aircraft accidents (IVW, 2003) (Eshati, 2006). Despite some tragedies, the main repercussions of maintenance errors are more likely to be felt at the bottom line than in injuries and fatalities. Maintenance errors cause large and continuing financial losses. General Electric estimates that each in-flight engine shutdown - for which maintenance error is the cause in 20-30 \% of all cases (Reason, 2003) - costs the airline somewhere in the region of $\$ 500000$.

The good news is that maintenance errors are not random. The vast majority of maintenance errors fall into well-defined clusters and recurrent patterns which are shaped mostly by task factors and situational characteristics (Reason and Hobbs, 2003). 
Maintenance errors can therefore be managed in the same way that any well-defined business risk can be managed. For this it has to be predicted where errors are most likely to occur so that preventative measures can be put in place.

A powerful tool in reducing maintenance errors is learning from the past. The problem is however that often maintenance organizations lack quality data that is of real added value to steer error reduction strategies. This is also the case for the operator under study (from this point onward referred to as Operator X). Occurrences are classified in a basic and incomprehensive manner. The basic classification of occurrences, in combination with the basic analysis techniques used, results in analyses that are incapable of being of real added value in understanding occurrences or steering error reduction strategies. The fact that effective error management on basis of database analysis is not possible formed the problem for this thesis.

The overall objective for this study is to learn more from occurrences based on database analyses. This abstract objective has been concretized into the two following concrete objectives:

1. Phase 1: Develop or select a maintenance error classification system (taxonomy) for Operator $X$, which can be integrated in the existing occurrence database software.

2. Phase 2: Analyze aircraft maintenance occurrences using the taxonomy from phase 1.

\section{Phase 1: Selecting an applicable error taxonomy}

Literature abounds with error taxonomies differing in levels of complexity and is dominated by different human error perspectives. Also area of application, focus, purpose and output differs between models (Lehto and Salvendy, 1998). From the practitioners point of view there often appears to be as many human error models and frameworks as there are people interested in the topic (Wiegmann and Shappel, 2001) In order to be able to see the wood for the trees, first, requirements, desirables and limitations for the new taxonomy were identified, based on the situation at Operator X. Using the requirements and the literature lessons, candidate error taxonomies and evaluation criteria that can be used to test the applicability of an error taxonomy against were selected. The evaluation criteria were applied by means of a desktop analysis and a practical test of the error taxonomies at the investigators. The final step was to select/develop the most applicable error taxonomy.

\section{Analysis}

An analysis of the occurrence management process at Operator $\mathrm{X}$ revealed the requirements and desirables for the taxonomy.

The principal requirements for the error taxonomy included:

- The error taxonomy should be able to describe occurrences comprehensively and accurately; the taxonomy should allow for the classification of occurrences on multiple aspects and distinguish accurately between various events. 
- The taxonomy has to be able to be integrated in the existing software framework in use at Operator X, meaning that it is not looked for heavy software packages, database systems, etc.

- The taxonomy has to be a well-known, validated taxonomy as easy communication with industry partners and authorities is important.

- The taxonomy should support the investigator during his investigation to identify human factor issues; this means the taxonomy should be practical and allow for detailed coding

- The taxonomy should capture information on occurrences in fixed coding options. This requirement has to ensure that the taxonomy can easily be used in the analysis and can deal with the large amount of occurrences per year.

These requirements were used to select the candidate taxonomies that were taken into account for this study.

In the last decade, the theories of James Reason have become the industry standard for explaining, understanding and classifying maintenance errors and occurrences. Reason's classification of unsafe acts is consistent with human information processing theories [McDonald, (2001)] and draws on the skill-rule-knowledge distinction of Rasmussen (1983) and the slip/mistake dichotomy of Norman (1981), but also includes violations as a distinct form of unsafe act.

Violations have not always been included in the classification of unsafe acts, though they form a significant problem in the aircraft maintenance industry. According to Reason, skill-based (unintended) unsafe acts take the form of slips and lapses, whereas intended action resulting in either rule-based or a knowledge-based mistake.

Another model of Reason that earned industry-wide recognition is his model of organizational accidents. This model is better known in the industry as the Swiss cheese model. The model is based on the notion that errors, or rather unsafe acts, are not random events but rather occur in response to causal factors, called latent failures in the model of Reason (1990). In most error taxonomies latent failures are referred to as contributing factors. One can find in literature a great range of potential error promoting factors, relating to almost all aspects of human performance but experience shows however that ultimately a relatively limited number of local factors appear over and over again in maintenance accidents and incidents [Boeing, (2001)]. These can be grouped as personal factors, organizational factors and environments \& workspace factors [McDonald, (2001)]. The theories of Rasmussen and Reason form the foundation of most cognitive error taxonomies found in literature.

Cognitive human error models may help to reveal fundamental error forms, so called error genotypes [Hobbs and Williamson, (2003)]. These models reveal the underlying cause of human errors and thereby can serve as powerful tools to assist in the understanding and prevention of human error. In most recent studies on maintenance errors, in which cognitive error models are used, it can be seen that the analysis concentrates on the following four aspects of an occurrence. 
1. Consequences: Consequences describe how the maintenance error affected operations or normal work processes. Examples are rework, damage or an operational effect such as a delay.

2. Maintenance error: A maintenance error describes the observable manifestation of an occurrence such as installation error, inspection error, repair error and documentation error.

3. Internal error: Internal errors describe how the mechanic's performance failed to achieve the desired result. Examples are slips \& lapse, knowledge-based error and violations.

4. Contributing factor: Contributing factors are those external factors that affect performance. Examples are task factors such new task or organizational factors such as overtime or lack of support from other departments or personal factors such a complacency.

Together, these four attributes of an occurrence are capable of sketching a complete picture of an event and distinguishing accurately one event from another.

For the aircraft maintenance industry, several cognitive models have been developed. Three validated and frequently used models were selected from literature as candidate error taxonomies for this study.

The oldest and most widely used taxonomy in the aircraft maintenance industry is Boeing's Maintenance Error Decision Aid (MEDA). MEDA allows coding on consequences and has an extensive list of maintenance error coding options, such as "panel not closed" and a rather extensive list of 72 contributing factors options including "inadequate knowledge", "fatigue", etc. MEDA is primarily designed as an investigation tool, but is also used for analysis purposes. In common with MEDA the Aircraft Dispatch and Maintenance Safety (ADAMS) system includes a large list of consequence, maintenance error and contributing factor options. ADAMS provides even more options than MEDA in all sections. In addition, ADAMS has separate sections for coding so called internal errors. Internal errors describe how the mechanic's performance failed to achieve the desired result. Internal errors can better enable the consideration of measures to reduce or mitigate errors, because the internal cause of the error can be analyzed [Shorrock, (2002), McDonald, (2001)]. Examples of internal error in the ADAMS form are "habit capture" or "memory failure".

The Human Factor Analysis Classification System Maintenance Extension (HFACS-ME) does not allow to code occurrences on consequences or maintenance errors, but focuses purely on the internal error and contributing factors of an occurrence.

The Sentinel taxonomy was added as this taxonomy was already present in the software framework at Operator $X$. The Sentinel taxonomy can be compared to the MEDA taxonomy, as it captures the same information on occurrences; only its structure differs. In Sentinel not only maintenance errors, but also non human-factors issues such as e.g. 
"bird strikes" can be coded. This makes the total amount of coding options much more extensive, though not all options are relevant for aircraft maintenance occurrences.

In order to be able to compare the four taxonomies evaluation criteria are needed. In literature no standardized set of evaluation criteria for testing the effectiveness of error taxonomies exists, however, the five criteria of Wiegmann and Shappell (2001) are frequently used:

- Reliability: the degree to which the taxonomy leads to consistent analyses between different users and with the same user over time.

- Comprehensiveness: the ability to discriminate and classify a comprehensive range of errors, influencing factors, etc., that together describe the occurrence accurately.

- Diagnosticity: the degree to which the taxanomy is capable of touching upon the root of the problem.

- Usability: the ease of use of the technique

- Validity: referring to theoretical validity, contextual validity but moreover the degree to which the taxonomy satisfies the other criteria.

\section{Design}

A desktop comparison and a practical test of the taxonomies at the investigators of Operator X was performed to determine how the taxonomies scored on the evaluation criteria. The desktop comparison revealed that only ADAMS captures the aspects consequences, maintenance error, internal error and contributing factors in separate sections and into most detail. This makes ADAMS the most comprehensive taxonomy. ADAMS, together with HFACS-ME scored also well on the area of diagnosticity as both collect information on both internal errors and contributing factors.

MEDA and Sentinel also collect all aspects but combine the contributing factor and internal error section. This apparent small difference has a significant impact as the test revealed that due to this internal errors are often not coded; internal factors are more difficult to identify - being internal - whereas external contributing factors are often more easier to identify - being external.

In the taxonomy the internal error options are often skipped as the investigator is not forced to code them; he can also select another contributing factor of which he is more certain. Besides, MEDA and Sentinel offer only a small list of internal error coding options. Nonetheless, the test revealed that MEDA was the preferred taxonomy in to be used during the investigation. Its wide-spread use throughout the aircraft maintenance industry, its user friendliness in the investigation, its logical structure and good balance between detail and flexibility were all characteristics that were very much appreciated by the investigators. The structure of MEDA, its compactness, make skimming through the taxonomy easy and coding options can be easily found. This favors its supportive function. MEDA could be used most reliably in the investigations and it can therefore be concluded that MEDA scored best on usability and reliability. 


\begin{tabular}{|l|l|}
\hline Internal error & Example \\
\hline Perceptual error & $\begin{array}{l}\text { Example: while performing an inspection a mechanic misses } \\
\text { obvious damage. }\end{array}$ \\
\hline Memory lapse & Example: rags left behind in the fuel tank. \\
\hline Slip & $\begin{array}{l}\text { Example: Without thinking, I moved to wipe oil with a rag. The rag } \\
\text { was ingested in the engine intake causing FOD (auto-motive } \\
\text { response) }\end{array}$ \\
\hline Rule based error & \begin{tabular}{l} 
Failure to apply unspoken rules of good practice.) \\
\hline Example: A mechanic did not check the position of the flap lever \\
before he pushed in a cockpit circuit breaker that provided \\
electrical power to a hydraulic pump. When the pump started, the \\
flaps began to retract automatically. This could have caused \\
damage to the aircraft or injured other workers.
\end{tabular} \\
\hline Knowledge-based error & $\begin{array}{l}\text { Example: A mechanic was carrying out a job for the first time, } \\
\text { misinterpreted the documentation and installed a part upside down }\end{array}$ \\
\hline Short-cut taken & $\begin{array}{l}\text { Example: A mechanic skips a step from the manual since he } \\
\text { knows he faster way to get the job done. }\end{array}$ \\
\hline System-induced violation & $\begin{array}{l}\text { Example: A mechanic due to perceived pressure omits an } \\
\text { inspection and signs off a task. }\end{array}$ \\
\hline Violation other & $\begin{array}{l}\text { Example: A mechanic intentionally breaks standing rules } \\
\text { disregarding the consequences. }\end{array}$ \\
\hline
\end{tabular}

TABLE 1: DEFINITIONS AND EXAMPLES OF INTERNAL ERROR SECTION OF THE ADAPTED MEDA TAXONOMY

Eventually it was chosen for an adapted MEDA taxonomy to be used in the future for classifying occurrences at Operator X. Adapted, because in order to improve the diagnosticity of the MEDA taxonomy an extra section, the internal error section, was added. Table 1 shows the coding options of this section and shows an example of each error type. The structure and coding options of this section were taken from ADAMS and HFACSME as these taxonomies scored better on this criteria. For the other sections the structure, format and coding options of the original MEDA form were taken; small adjustments were made, such as extra coding options, to make the taxonomy more applicable for the operator under study. The adapted MEDA taxonomy now captures information on occurrences in four sections: consequences, maintenance error, contributing factors and an internal error section. General information, referring to place, date, time, a/c type etc, is already captured in the Sentinel system outside of the taxonomy so this section was not included in the taxonomy. Some coding options were added however, such as location of error, phase of operation and where the error manifested itself. Again, this was done to make the taxonomy more applicable and meaningful for this particular operator.

The primary reasons why the adapted MEDA taxonomy was preferred over the other 
taxonomies were:

HFACS-ME was considered very unpractical in the investigation as maintenance errors and consequences cannot be coded in the taxonomy resulting in an incomplete story of the occurrence. Also the terms used on the form were considered too academic for the practical maintenance environment. HFACSME was also considered very inflexible as incomplete investigations, for example an investigation that happened too long ago to still be possible to determine causes, cannot be coded at all in the taxonomy resulting in loss of information.

ADAMS was considered very comprehensive due to its level of detail and many sections. However, its length, 16 pages, compared to MEDA's five pages was considered unpractical and frustrating in situations where occurrence details were lacking. Also, its enormous level of detail makes reliable coding often impossible. This also applied to the internal error section; the amount of coding options was simply too large. Another disadvantage of ADAMS is that ADAMS is far less popular in the industry than MEDA and HFACS-ME. This makes exchanging investigation results with authorities and other companies more difficult.

Sentinel was considered a worse version of the MEDA taxonomy. Its coding options are similar in the end but navigating through the taxonomy is a real puzzle as its structure is considerable worse than the structure of MEDA.

The added value of the adapted MEDA taxonomy over the previous classification system(s) in use at Operator X stems from the following:

The adapted MEDA taxonomy is of better support during the investigation to identify human factor issues as the taxonomy focuses on multiple aspects of an occurrence such as consequence, maintenance error, internal error, contributing factor, location of error etc. Also the increased level of detail in all sections forces the investigator to dig deeper resulting in more detailed information for the analysis.

The adapted MEDA taxonomy is more value during the analysis as occurrences can be better distinguished from one another through the different sections. The various layers, adding more or less detail, allow for robust comparison which is beneficial as it is looked for similarities in analyses and not differences between occurrences.

The adapted MEDA taxonomy better reflects the company's vision on its "Just Culture"; in the adapted MEDA taxonomy honest errors can be better distinguished from systeminduced violations and violations.

\section{Phase 2: the analysis of maintenance occurrences}

\section{Method}

The study population for phase 2 consisted of occurrence investigation reports dated between January 2007 and September 2008, due to a maintenance error and investigated by either the investigator from one of the base maintenance hangars or the investigator of the line maintenance station of the operator. 
These three criteria ensured for a sufficient amount of reports for the analysis on maintenance errors. Investigation reports further back were not taken into account due to the limited timeframe for this analysis and because reports further back in time would likely deteriorate the reliability of the analysis as they are less comprehensive and detailed. The occurrences were coded using the taxonomy developed in phase 1.

For the analysis not the most detailed level of the classification system developed in phase 1 was used. The comprehensive list of options in all sections provides useful guidance for investigators in the investigation on a case by case basis but is less useful for database analysis. By placing occurrences into a large number of categories differences are emphasized and similarities are obscured. A more robust, less detailed classification system will be of more benefit in the analysis and lead to better interpretable results (Hobbs and Williamson, 2003). If more information is needed on a specific robust category, the classification system allows zooming in on the more detailed levels.

An occurrence can contain multiple events and one event can be due to several contributing factors. To capture this in the analysis, multiple coding options in the same section could be selected for one occurrence. For example, an installation error that was also not noticed during inspection was coded as "installation error "AND "inspection error". Each maintenance error (each event) was then linked to its appropriate internal error. If the "installation error" was for example due to a "knowledge-based mistake" these two were linked and if the "inspection error" was due to a "perceptual error" these two were also linked separately. The contributing factors leading up to the specific internal error were also linked separately to each event.

The focus of the analysis was on associations between various attributes of an occurrence. In most studies on aircraft maintenance events, the various attributes of an occurrence are analyzed separately in unlinked tables. A major limitation of this type of research is that lessons learned in one domain might not generalize to other domain [(Hobbs and Williamson, 2003)]. For example, concluding from one table that slips and lapses are the most frequent error type, and concluding from another table that fatigue is the most predominant contributing factor does not necessarily mean that slips and lapses are affected by fatigue. Continuing with the example of internal errors and contributing factors, it is assumed in most studies for the sake of simplicity, that all factors increase the prevalence of errors equally. However, it seems likely, that specific contributing factors would be associated with particular forms of human fallibility. At the very least, it might be expected that conditions or factors that promote error of automatic performance (such as slips) would be different from those that promote mistakes involving controlled processing (such as rule-based and knowledge based errors) [Hobbs and Williamson, (2003)]. For error management purposes, that is management aimed at reducing errors or containing its negative consequences, it is most interesting to look at these interactions between the various aspects of an occurrence [Williamson and Hobbs, (2003)]. Such information has several important uses. For one, occurrence prevention strategies can be targeted at, for example, key factors that promote a certain error. 
Occurrences were analyzed by using three analysis techniques: frequency counts \& percentages, stacked tables and correspondence analysis.

"Frequency counts" is the most common method of analyzing occurrences used in literature and a good method to get an overview of the problern areas within the occurrence data.

"Stacked tables" is a relative straight forward way of displaying the association between variables. In a stacked table it is shown what part of variable 1 is due to variable 2; each bar (variable 1) is divided into parts (variable 2)

Arguable the most interesting analysis technique used in this study was "Correspondence analysis" (CA). CA is a technique well suited to summarily describe relationships among categorical variables. It is an exploratory procedure that converts complex data tables into a visual form that is easier to interpret. The technique is inductive, a search strategy for underlying structure within a data set. Correspondence analysis is a method especially applicable for analyses of large contingency tables. The technique is a tool to analyze the association between two or more categorical variable by representing the categories of the variables as points in a low-dimensional space. Categories with similar distributions will be represented as points that are close in space, and categories that have very dissimilar distributions will be positioned far apart.

The technique requires no assumptions about the data, other than that values are not assumed negative. (Clausen, 1998).Correspondence analysis can be found in most commercial statistical software packages. This paper however is not about correspondence analysis but rather its application. For interpretation purposes it is sufficient to focus on the distances between points: the closer two points are located in the CA plot, the more similar profiles they have, the closer they are associated.

In total 858 different occurrence reports were reclassified and analyzed for this study. Of these, 809 investigation reports contained usable data. Not all reports contained usable data. Some did not contain an occurrence at all or did not concern a maintenance event.

\section{Nature of occurrences}

In total 940 maintenance errors were identified. The most commonly identified maintenance error was an "installation error". Installation errors were identified in a quarter of all occurrences. Typical examples of installation errors are "part is not installed", "wrong part installed" or "correct part is wrong installed". The second most frequent encountered maintenance error is a "documentation error" for example the situation where an aircraft is released for service without completing the required documentation. "Test/inspection" errors typically concerned "a not performed inspection" or a "failure to notice a visible fault" (e.g. damage, a lost tool).

Table 2 lists the identified maintenance errors. 


\begin{tabular}{|l|l|l|}
\hline Error type & Frequency & Percentage \\
\hline Installation error & 234 & 25 \\
\hline Documentation error & 136 & 14 \\
\hline Test/Inspection error & 101 & 11 \\
\hline Servicing error & 67 & 7 \\
\hline FOD & 56 & 6 \\
\hline A/C Damage error & 54 & 6 \\
\hline Removal error & 54 & 6 \\
\hline No ME & 43 & 5 \\
\hline Other & 41 & 4 \\
\hline Unknown Me & 40 & 4 \\
\hline Secure/Seal & 28 & 3 \\
\hline Repair error & 25 & 3 \\
\hline Not reported & 25 & 3 \\
\hline System not activated & 23 & 2 \\
\hline Test not performed & 13 & 1 \\
\hline Total & 940 & 100 \\
\hline
\end{tabular}

TABLE 2: OVERVIEW OF MAINTENANCE ERRORS

Table 3 list the identified consequences. In total 878 consequences were identified. The most commonly noted occurrence outcome was "none"; often an occurrence is reported even if nothing really happened. "Rework" and "Damage" are the second and third most identified occurrence outcomes. Arguably the most interesting consequences are the operational effects as occurrences with an operational effect are likely to have the greatest impact on safety and economics. Two different operational effects are distinguished: "severe" and "other/minor". Examples of severe operational effects are delays, cancellations, air turn backs and in-flight engine shutdowns. A other minor operational effect typically concerned an occurrence where the crew reported hinder during operation - for example vibration, noise, unusable seat I toilet.

\begin{tabular}{|l|l|l|}
\hline Consequences & Frequency & Percentage \\
\hline None & 316 & 36 \\
\hline Rework & 173 & 19,7 \\
\hline A/C Damage event & 133 & 15,1 \\
\hline Operational effect (other/minor) & 112 & 12,8 \\
\hline Operational effect (severe) & 102 & 11,6 \\
\hline Other & 42 & 4,8 \\
\hline Total & 878 & 100 \\
\hline
\end{tabular}

TABLE 3: OVERVIEW OF CONSEQUENCES

Table 4 presents an overview of the internal errors. In total 940 internal errors were identified. "Slips" are the most commonly identified internal error. This result strokes with consensus in literature (Hobbs, 2003). The second most commonly identified category is "unknown". The reason why in many investigation reports the internal error could not be retrieved is because investigators at were not used to identifying internal 
errors. Although "violations" appear as the least frequent categories, combined they add up to $14 \%$ making them still significant categories.

\begin{tabular}{|l|l|l|}
\hline Internal error & Frequency & Percentage \\
\hline Slip & 236 & 25 \\
\hline Unkown & 175 & 19 \\
\hline Knowledge based error & 110 & 12 \\
\hline Lapse & 107 & 11 \\
\hline Mischance \& none & 99 & 11 \\
\hline Rule-based error & 72 & 8 \\
\hline Routine violation & 58 & 6 \\
\hline Violation other & 42 & 4 \\
\hline Short-cut taken & 41 & 4 \\
\hline Total & 940 & 100 \\
\hline
\end{tabular}

TABLE 4: OVERVIEW OF INTERNAL ERRORS

Table 5 lists the identified contributing factors. In total 1490 contributing factors were identified. The most frequent identified contributing factor is "unknown"; many investigation reports were too brief to make assessments to its error promoting factors. "Mental \& physical state" is the second most commonly identified contributing factor. This typically refers to morale issues such as "complacency", "frustration" or "uncertainty".

Although personal factors are most frequently identified it is not legitimate to conclude that most maintenance errors are due to factors under control of the mechanic; all the other categories in Table 5 such as task factors, task support factors or organizational factors are not under direct control of the mechanic and add up to over 50\% combined. Nonetheless, this result differs from a study performed by Boeing (2001). Boeing states that only $10-20 \%$ of the error promoting factors are under direct control of the mechanic and $80 \%$ are under management control.

\section{Associations between the variables}

The correspondence analysis biplot shown in Figure 1 displays the relationships between maintenance errors and consequences. It is apparent from this figure that maintenance errors are not evenly distributed across consequences. Three clusters of errors and consequences emerged:

1. "Operational effect (OE)" and "rework" clustered together with "installation errors", "servicing errors" and "repair errors" and "test \& inspection errors".

2. The consequence "None" is associated with "documentation errors".

3. The consequence "AlC Damage event" is associated with "damage \& FOD errors". 


\begin{tabular}{|l|r|r|}
\hline \multicolumn{1}{|c|}{ Contributing factors } & Frequency & Percentage \\
\hline Unkown & 277 & 19 \\
\hline Mental \& physical state & 254 & 17 \\
\hline Skills \& knowledge & 218 & 15 \\
\hline Information & 145 & 10 \\
\hline Procedure issues & 84 & 6 \\
\hline Communication \& Cooperation & 75 & 5 \\
\hline Job/Task & 66 & 4 \\
\hline Design & 60 & 4 \\
\hline Leadership & 53 & 4 \\
\hline Training & 52 & 3 \\
\hline Environment \& Workspace & 43 & 3 \\
\hline Workgroup normal practice & 40 & 3 \\
\hline Tools \& Equipment & 36 & 2 \\
\hline Time management & 25 & 2 \\
\hline Support from other departments & 25 & 2 \\
\hline Company policy & 23 & 2 \\
\hline Staff problems & 14 & 1 \\
\hline Total & 1490 & 100 \\
\hline
\end{tabular}

TABLE 5: OVERVIEW OF CONTRIBUTING FACTORS

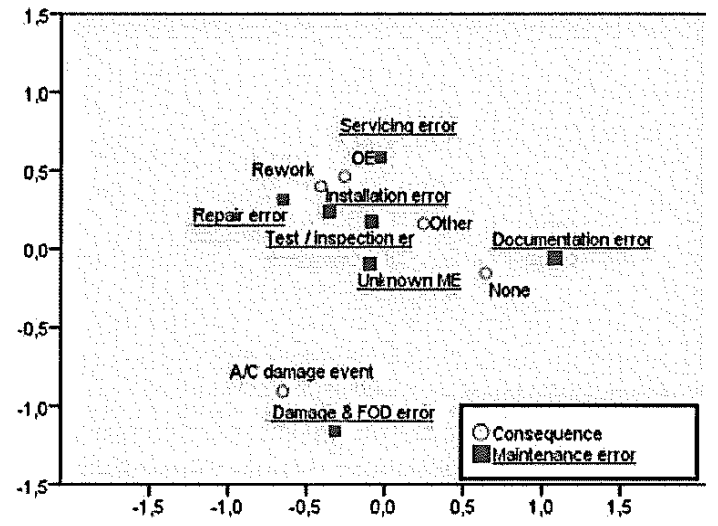

\section{FIGURE 1: MAINTENANCE ERRORS AND CONSEQUENCES}

The correspondence analysis biplot of Figure 2 displays the relationship between maintenance errors and internal errors. Several clusters emerge:

1. "Documentation errors" are associated with "violations". "Violations" are to a lesser extent associated with "test \& inspection errors". 
2. $\quad$ "Installation errors" and "servicing errors" are clustered together with "slips \& lapses", "knowledge-based errors", "rule-based errors" and "unknown internal error".

3. $\quad$ "Damage \& FOD" errors are most associated with slips and lapses.

4. $\quad$ "Servicing errors" are mostly associated with rule-based errors, knowledge-based errors, slips and lapses and mischance.

5. $\quad$ "Test I inspection errors" are not clustered together with any internal error but show strongest association with slips and lapses and to a lesser extent "violations".

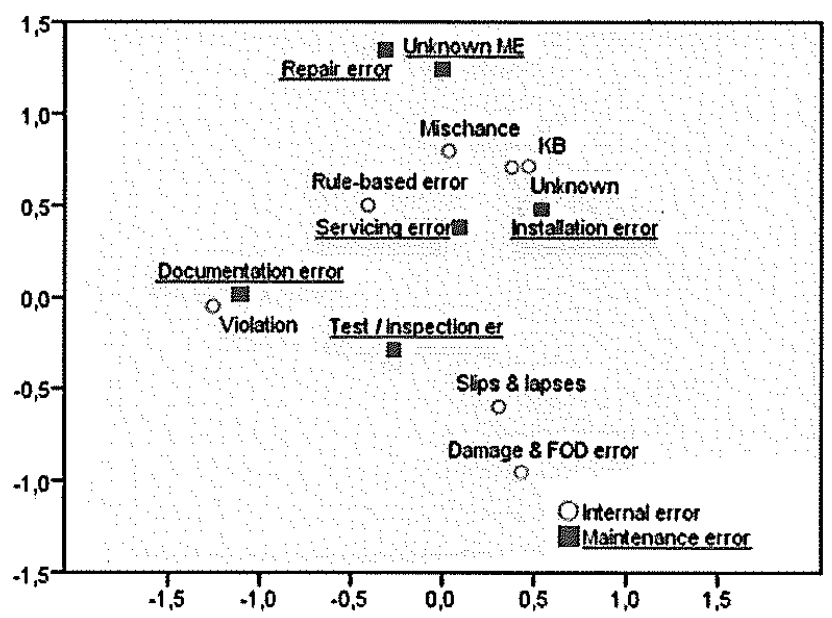

FIGURE 2. MAINTENANCE ERRORS AND INTERNAL ERRORS

The CA biplot of Figure 3 displays the relationship between internal errors and contributing factors. In this plot, the categories of "mischance" and "unknown" for the internal errors have been made supplemental. Supplementary points is a useful property of CA that allows for better interpretation when points appear clustered together in the origin due to outlying points; other points are pressed together due to the outliers. "Unknown" and "mischance" were such outliers causing the other points to appear close together near the origin. By displaying these two categories as supplementary points it is possible to better interpret the other points as $\mathrm{CA}$ is now better able to display the variance in the other profiles. Drawback is that "unknown" and "mischance" can now not be interpreted. 


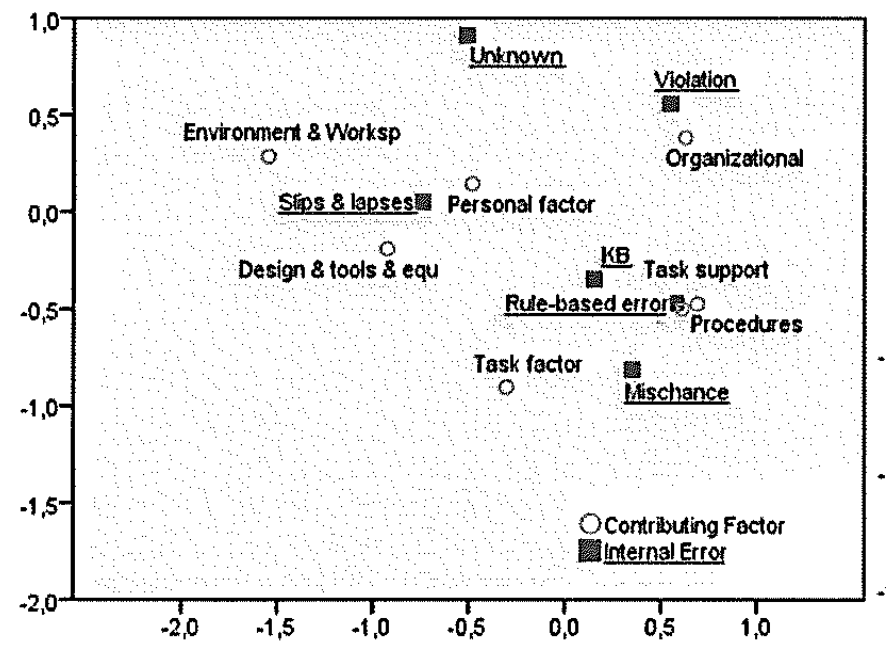

FIGURE 3. INTERNAL ERRORS AND CONTRIBUTING FACTORS

In the CA biplot several clusters appear:

1. The most common error type, "slips \& lapses", was most associated with "personal factors", "environment \& workspace", "design, tools and equipment", and "organizational factors",

2. "Knowledge-based errors" and "rule-based errors" can be found near the centre of the plot. "Knowledge-based errors" and to a lesser extent "rule-based errors", show association with almost all contributing factors except environment \& workspace.

3. "Violations" are most associated with organizational and personal factors and to a lesser extent with task support and task factors.

Besides associations between the four main variables of the taxonomy (consequences, maintenance errors, internal errors and contributing factors), also associations between other aspects of an occurrence can be displayed, for example, associations between maintenance errors and "the location of error".

Figure 4 displays the CA biplot of maintenance errors and location of error. Location of error is not one the 'core' sections of the adapted MEDA taxonomy but nevertheless valuable lessons can be learned by involving location of error in the analysis, The associations that emerged are:

1. "Hangar A" and "hangar B" (base maintenance hangars) are clustered with "installation errors", "test/inspection errors" and "damage errors".

2. Hangar $\mathrm{A}$ is more associated with "damage errors" and "hangar B" more with "installation errors". 


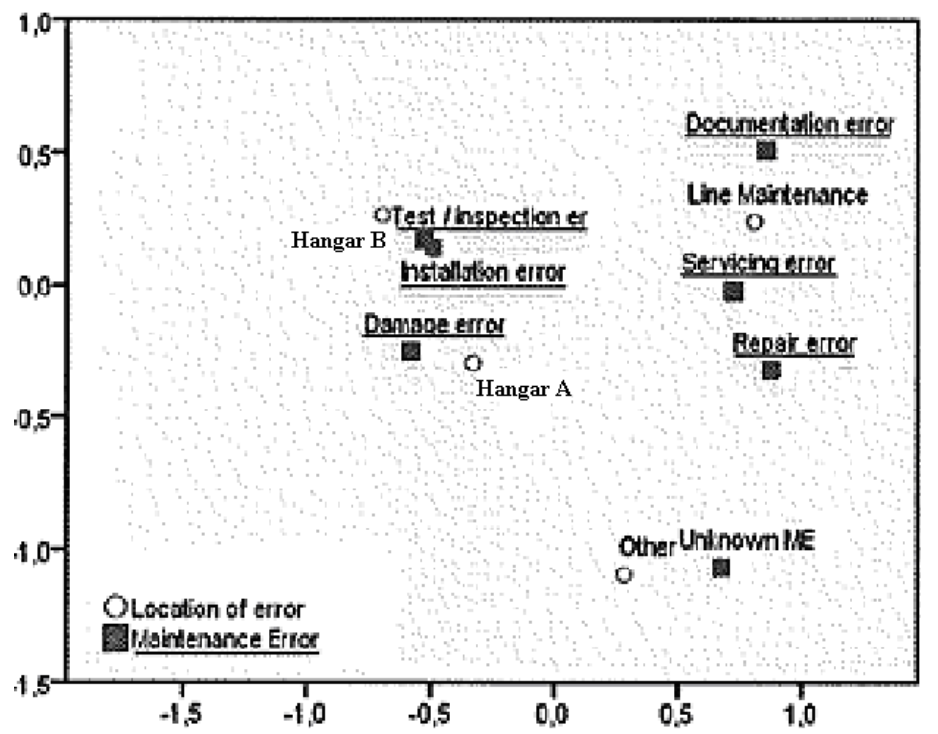

FIGURE 4. MAINTENANCE ERROR AND STATION OF ERROR

\section{Discussion}

With the amount of information that can be collected in an investigation and the above presented analysis techniques many different figures and tables can be developed that provide insight in the occurrence data. The above figures are of course just a subset of the total amount of figures and plots that can be made. There is not one figure or table that tells the whole story. It is therefore a necessity to make various plots and interpret them simultaneously in order to gain insight in the occurrences.

The results have emphasized the primary place of human behavior in the development of maintenance occurrences. This analysis provides evidence from safety related occurrences at the operator that clear associations exist between the various aspects of an occurrence. The design and set up of the current study does not allow causal inferences to be drawn from the patterns of consequences, maintenance errors, internal errors, contributing factors and location of error. Nevertheless, the associations among the several categorical variables suggest several targets for intervention. For example, the close relationship between "installation errors" and "operational effect" suggest that a useful way to address this consequence would be to target the installation errors and the internal errors and contributing factors that promote them - most notably "slips \& lapses" which were frequently associated with "task factors", "personal factors", "organizational factors" and "tools and equipment".

More generally, the specific links between the various aspects of an occurrence provide guidance for safety managers seeking to reduce the incidence of human error in the aircraft maintenance tasks. 


\subsection{Added value and conclusion for phase 2}

The added value of the analysis is that now discussions about occurrences can be backed up with factual data. In the past the factual discussion stalled at the assessment that, for example, installation errors are the most common maintenance error as occurrences were nor classified nor analyzed on various aspects. The use of different analysis techniques enhances the understanding of occurrences, their causes, effect, where they are especially apparent, etc. The links between the various aspects of an occurrence provide guidance for the safety managers seeking to reduce the incidence of human error in the aircraft maintenance tasks.

On basis of this analysis it was not recommended to the operator under study to immediately take actions; all field studies suffer to a greater or lesser extent from the possibility that the data reflects biases. In this study, occurrences were classified based on finished investigation reports (post hoc). Occurrences could not be reinvestigated if some information was lacking in the reports. It is possible that certain circumstances or errors have been misinterpreted or were simply not indicated in the reports. The results will become more reliable and hence improve once the taxonomy is effectively used. This analysis should be seen a good starting point; to see what is possible and what direction the analysis might steer to.

In this paper the process and result for selecting an applicable error taxonomy for a particular air operator was discussed as were the method and results of an analysis of maintenance occurrences at this operator.

The selection process for the error taxonomy involved various stakeholders of the error taxonomy. Investigators, safety officers and management were all involved so that in the end the most applicable error taxonomy for all parties was chosen. Eventually an adapted version of the MEDA taxonomy was chosen. The adapted MEDA taxonomy is better capable of describing occurrences comprehensive and accurately and is of better support in the investigation to identify (for the analysis) relevant human factor issues. It is recommended that investigators start using the adapted MEDA taxonomy and that this taxonomy is implemented in existing software frameworks. It was shown that the error taxonomy can be used effectively in the analysis of occurrences. Also, it was shown that various statistical analysis techniques such as frequency counts, stacked tables and CA plots are a powerful in gaining insight in occurrence data. The analysis showed also several interesting links in the occurrence data between consequences, maintenance errors, internal errors, contributing factors and location of error. For example, the close relationship between "installation errors" and "operational effect" suggest that a useful way to address this consequence would be to target the installation errors and the internal errors and contributing factors that promote them - most notably "slips \& lapses" which were frequently associated with "task factors", "personal factors", "organizational factors" and "tools and equipment'. However, it is noted that these results might reflect biases as occurrences were classified post hoc without redoing the investigation. It is possible that information in the reports was lacking or has been misinterpreted.

It is recommended that air operators start using various analysis techniques such as stacked tables and CA plots, as these techniques will improve the understanding of maintenance errors. 


\section{References}

Boeing, (2001), Maintenance Error Decision Aid (MEDA) User guide.

Clausen, S.E., (1998), Applied correspondence analysis. Sage: Thousand Oaks.

Hobbs, A., Williamson, A. (2003), Associations between errors and contributing factors in aircraft maintenance. Human Factors, Vol 45, No.2, Summer 2003.

Hobbs A. (2001), The links between errors and error-producing conditions in aircraft maintenance.

Inspectie Verkeer en Waterstaat, (IVW), (2003), Veiligheidsstatistieken luchtvaart. Civil Aviation Safety data.

Lehto, M., Salvendy, G. (1999): Models of accident causation and their application: Review and reappraisal. Journal of Engineering and Technology Management,173-205.

Norman, D.A., (1981), The categorization of action slips. Psychological Review, 88, 1-15.

McDonald, N. et al (2000), Human-Centred Management Guide for Aircraft Maintenance. Appendices, European Community ADAMS Project.

Rasmussen, J., (1983), Skills, rules and knowledge: Signals, signs and symbols, and other distinctions in human performance models. IEEE Transactions on Systems, Man, and Cybernetics, SMC-13(3): 257-266

Reason, J., (1990), Human Error. Cambridge University Press, Cambridge.

Reason, J. Hobbs, A., (2003), Managing Maintenance Error, Ashgate Publishing Company.

Eshati, S., (2006), Critical Analysis of Maintenance Error Decision Aid (MEDA

and Human Factors Analysis and Classification System (HFACSME) in Aircraft Maintenance. School of Engineering, Cranfield University.

Shorrock, S.T., Kirwan, B., (2002), Development and application of a human error identification tool for air traffic control. Applied Ergonomics, 33, 319-336.

Wiegmann, D.A., Shapell, S.A., Human Error Perspectives in Aviation. The International Journal of Aviation Psychology, 11(4),341-357.

Choudry, M. C., F. Dongping, et al. (2007). "The nature of safety culture: A survey of the state-of-the-art." Safety Science 45: 993-1012.

Diaz-Cabrera, D., E. Hernandez-Fernaud, et al. (2007). "An evaluation of a new instrument to measure organisational safety culture values and practices." Accident Analysis and Prevention 39(6): 1202-1211.

Glendon, I., E. McKenna, et al. (2006). Human Safety and Risk management, CRC Press.

Guldenmund, F. W. (2000). "The nature of safety culture: A review of theory and research." Safety Science 34(1-3): 215-257.

HSE (2002). Human Factors Briefing Note. H. a. S. Executive, Health and Safety Executive. 7.

Hudson, P. (2001). Aviation Safety Culture, Centre for Safety Science, Leiden University.

Hudson, P. (2007). "Implementing a safety culture in a major multi-national." Safety Science 45(6): 697-722.

Kempen, P. M. and J. A. Keizer (2000). Advieskunde voor praktijkstages, Noordhoff Uitgevers B.V.

Lafrenière, P. J. (2006). Safety Management System Implementation in the Nuclear Power Industry: a collaborative effort. SMS Operators Meeting, Montreal, Quebec.

Reason, J. (1998). "Achieving a safe culture: Theory and practice." Work and Stress 12(3): 293-306.

Wiegmann, D. A., H. Zhang, et al. (2002). Safety culture: A Synthesis of Safety Culture and Safety Climate Research, Aviation Research Lab institute of Aviation. 